\title{
Optimal and Near-Optimal Pairs for the Estimation of Effects in 2-level Choice Experiments
}

\author{
Deborah J. Street \\ Department of Mathematical Sciences, \\ University of Technology, Sydney. \\ Leonie Burgess \\ Department of Mathematical Sciences, \\ University of Technology, Sydney.
}

\begin{abstract}
This paper gives constructions for optimal and near-optimal sets of pairs for the estimation of main effects, and for the estimation of main effects and two factor interactions, in forced choice experiments in which all attributes have two levels. The number of pairs is much smaller than that in many previously constructed optimal 2-level choice experiments.
\end{abstract}

AMS Subject Classification: Primary 62J15; Secondary 62K05.

Key words: Paired comparison; Bradley-Terry model; Optimal designs; Factorial design; Orthogonal main effect plan.

\section{Introduction}

Since proposed by Louviere and Woodworth (1983), interest in designed choice experiments has steadily increased, and their use in marketing, transport, environmental, resource economics and other fields has grown substantially. Despite progress in understanding how to design multiple choice experiments that are consistent with certain types of probabilistic discrete choice models (eg, multinomial logit regression models), there is still much to learn about the efficiency properties of various candidate designs. Work on determining the structure of the optimal designs has been carried out by Berkum (1987a, b; 1989), Offen and Littell (1987), Street, Bunch and Moore (2001) and Grossman, Holling and Schwabe (2002), amongst others.

This paper investigates the efficiency of various small sets of choice pairs in a setting in which estimates for main effects, or main effects and two factor interactions, are required and respondents must choose one of the two options presented in each choice set. 
The ideas in Berkum (1987a, b) are extended in Berkum (1989) to develop smaller sets of pairs that are optimal and near-optimal for the estimation of a quadratic model. Since the model to be estimated is quadratic, the attributes are quantitative and each has at least three levels. Thus these designs are not appropriate to the situation discussed in this paper.

Street, Bunch and Moore (2001) established the form of the optimal paired comparison design when there are $k$ attributes (or factors), each with two levels (or settings), and the set of possible choice pairs is restricted so that each pair of treatment combinations in which there are $i$ attributes with different levels appears equally often. If all the possible treatment combinations are to be used to generate the pairs (that is, the pairs are to be based on the complete factorial) then the optimal design for the estimation of main effects is the design consisting of all the foldover pairs. (One treatment combination is said to be the foldover of another if the second one is obtained from the first by changing all 0's to 1's and all 1's to 0's.) An example of the foldover pairs with $k=4$ appears in Table 1. The information matrix for these optimal designs for estimating main effects was shown to be diagonal and to have determinant $\left[\frac{1}{2^{k}}\right]^{k}$.

$\begin{array}{llllllll}\{(0 & 0 & 0 & 0), & (1 & 1 & 1 & 1)\} \\ \{(0 & 0 & 0 & 1), & (1 & 1 & 1 & 0)\} \\ \{(0 & 0 & 1 & 0), & (1 & 1 & 0 & 1)\} \\ \{(0 & 0 & 1 & 1), & (1 & 1 & 0 & 0)\} \\ \{(0 & 1 & 0 & 0), & (1 & 0 & 1 & 1)\} \\ \{(0 & 1 & 0 & 1), & (1 & 0 & 1 & 0)\} \\ \{(0 & 1 & 1 & 0), & (1 & 0 & 0 & 1)\} \\ \{(0 & 1 & 1 & 1), & (1 & 0 & 0 & 0)\}\end{array}$

Table 1: The fold-over pairs with $k=4$

In the same setting Street, Bunch and Moore (2001) also established that the optimal designs for the estimation of main effects and two factor interactions were those with all pairs with $(k+1) / 2$ attributes different (when $k$ is odd). For even $k$ the optimal designs were shown to be those with all pairs with $k / 2$ or $k / 2+1$ attributes different. The information matrix, $C$, for estimating main effects and two factor interactions using these designs was shown to be diagonal and to have determinant

$$
\operatorname{det}(C)= \begin{cases}{\left[\frac{k+1}{k 2^{k+1}}\right]^{k+\frac{k(k-1)}{2}},} & \text { when } k \text { is odd, } \\ {\left[\frac{k+2}{(k+1) 2^{k+1}}\right]^{k+\frac{k(k-1)}{2}},} & \text { when } k \text { is even. }\end{cases}
$$

The same result appeared in Berkum (1987a) using a different method of proof.

Thus any design that is proposed for estimating either main effects or main effects and two factor interactions can be compared to these optimal designs. In general the $D$-efficiency of any design is given by $\left(\frac{\operatorname{det}(C)}{\operatorname{det}\left(C_{\text {optimal }}\right)}\right)^{1 / p}$ where $p$ is 
the number of parameters that have to be estimated in the model. For designs that estimate main effects, $p=k$. For designs that estimate both main effects and two factor interactions, $p=(k+k(k-1) / 2)$.

This gives one way of comparing designs. Note that diagonal information matrices are preferable since diagonal matrices mean uncorrelated estimates. The constructions given later usually result in diagonal information matrices.

In the next section we recall a number of results about fractional factorial designs and about the information matrix for a choice experiment. In the remainder of this paper we will investigate the $D$-efficiency of various designs, both those that have been routinely used by marketers and some small designs that we are proposing as alternatives to the very large designs that arise from the results in Berkum (1987a, b) and Street, Bunch and Moore (2001).

\section{Preliminary Results}

We use $2^{k-p}$ to denote a $\frac{1}{2^{p}}$ fraction of a $2^{k}$ factorial design. A fraction is said to be regular if it can be described by the solution space to a set of $p$ independent binary equations. For instance, the solutions to the binary equation $x_{1}+x_{2}+x_{3}=0$ are the $8=2^{4-1}$ treatment combinations in Table $2(\mathrm{a})$.

$\begin{array}{llll}0 & 0 & 0 & 0 \\ 0 & 0 & 0 & 1 \\ 0 & 1 & 1 & 0 \\ 0 & 1 & 1 & 1 \\ 1 & 0 & 1 & 0 \\ 1 & 0 & 1 & 1 \\ 1 & 1 & 0 & 0 \\ 1 & 1 & 0 & 1\end{array}$

(a) Resolution 3

$\begin{array}{llll}0 & 0 & 0 & 0 \\ 0 & 0 & 1 & 1 \\ 0 & 1 & 0 & 1 \\ 0 & 1 & 1 & 0 \\ 1 & 0 & 0 & 1 \\ 1 & 0 & 1 & 0 \\ 1 & 1 & 0 & 0 \\ 1 & 1 & 1 & 1\end{array}$

(b) Resolution 4

Table 2: OMEPs with $k=4$

A particular class of fractional factorial designs are the orthogonal main effect plans. Recall that a $k$ attribute, $n$ run, two-level orthogonal main effect plan (OMEP) is an $n$ by $k$ array of 0 's and 1's such that each column contains the same number of 0 's as of 1's and all main effects are independently estimable. Such an array is said to have resolution 3. The design in Table 2(a) is an example of an OMEP with $k=4$ and $n=8$.

Subsequently we will need designs of resolution 4 and 5. A design is said to have resolution 4 if two-factor interactions may be confounded with each other but never with main effects. An example of a design of resolution 4 with $k=4$ and $n=8$ appears in Table 2(b). A design is said to have resolution 5 if main effects and two-factor interactions can be independently estimated. A design of resolution $r$ is also a design of resolution $r-1$. 
Note that if we represent the two levels of an OMEP by -1 and 1 then the inner product of any two columns of the OMEP is 0 .

For a regular fractional factorial design there is a link between the resolution of the fractional factorial and the equations that define it. Consider the $p$ independent binary equations that define a regular $2^{k-p}$ design. Calculate all the linear combinations of these binary equations. If each of the original equations and each of the linear combinations have at least $r$ non-zero coefficients then the $2^{k-p}$ fraction is of resolution $r$. For instance the design in Table 2(b) is the solutions to the binary equation $x_{1}+x_{2}+x_{3}+x_{4}=0$.

The following results about the information matrix will be helpful subsequently.

We let the information matrix of the set of choice pairs be denoted by $C$ and, following El-Helbawy, Ahmed and Alharbey (1994) and Street, Bunch and Moore (2001), we let $\Lambda$ be the matrix of second derivatives of the log-likelihood function (assuming the Bradley-Terry model is used). With this model, an assumption that all the $\pi_{i}$ are equal and this definition of $\Lambda$, we find that $\Lambda_{i j}, i \neq j$, is one-quarter of the negative of the proportion of pairs in the choice experiment that compare treatments $i$ and $j$, where we have ordered the treatment combinations in some fixed, but arbitrary, way. Note that here we are using $i$ and $j$ to refer to $k$-tuples of 0's and 1's. The diagonal elements of $\Lambda$ are such that the row and column sums of $\Lambda$ are 0 . The $\Lambda$ matrix for the pairs in Table 1 (where we use lexicographic order for the treatment combinations, that is, 0000, 0001, 0010, 0011 and so on) is $I_{16}-R_{16}$, where $I_{n}$ is the identity matrix of order $n$ and $R_{n}$ is an order $\mathrm{n}$ matrix with 1 's on the backdiagonal and 0's elsewhere.

Let $B_{m c}$ be the normalised contrast matrix for main effects in the complete factorial.

With these definitions it can be shown that the information matrix for estimating main effects, $C_{m}=B_{m c} \Lambda B_{m c}^{T}$, although if a fractional design is used some rows and columns of $\Lambda$ will be 0 . When comparing two, or more, designs, we assume that all treatment combinations could have been involved in the design. Otherwise we are implicitly restricting attention to only those designs that can be obtained from a fraction of the treatment combinations. Designs constructed from fractions may be quite uninformative compared to ones obtained from the complete design.

In the next section we will investigate the $D$-efficiency of two classical constructions for choice experiments. In the section following we will look at the $D$-efficiency of designs obtained from using the pairs that result by adding one, or more, vectors to each of the treatments in a regular fraction.

\section{Two Classical Constructions}

The two constructions that we consider in this section are used frequently; see Louviere (1988), Kuhfeld (2000) or Louviere, Hensher and Swait (2000), for example.

The first construction is found as Option 3 in Appendix A5 of Louviere, 
Hensher and Swait (2000). No formal proof of the properties of these designs is given there.

Construction 1 To construct a set of pairs to compare products with $k$ attributes, first construct a regular orthogonal main effect plan with $k$ attributes. From each row of this OMEP obtain a choice pair by pairing the row with its foldover. If any pair appears twice then the duplicate choice set is omitted. Thus each treatment combination, and each pair, appears only once in the final set of choice pairs. The design has a diagonal information matrix and a D-efficiency of $100 \%$ for estimating main effects.

\section{Proof of Construction 1}

Assume that all the equations that define the OMEP have an even number of coefficients. Let $\sum_{i} \eta_{i} x_{i}=0$ be one of these equations. Then $\sum_{i} \eta_{i}=$ 0 . If $\left(a_{1}, a_{2}, \ldots, a_{k}\right)$ is a solution of this equation then $\sum_{i} \eta_{i} a_{i}=0$. Hence $\sum_{i} \eta_{i}\left(1-a_{i}\right)=\sum_{i} \eta_{i}-\sum_{i} \eta_{i} a_{i}=0$ and so the OMEP consists of foldover pairs.

Let the levels for the factors be -1 and 1 and let $A$ denote the $n \times k$ array for the OMEP. Since $A$ consists of fold-over pairs, we can represent $A$ as $\left[\begin{array}{c}A_{1} \\ -A_{1}\end{array}\right]$. Thus $A^{T} A=n I_{k}=2 A_{1}^{T} A_{1}$. We can write the pairs as $\left(A_{1},-A_{1}\right)$. Then the $B$ matrix for the treatment combinations that are actually involved in the design is $B_{m f}=\frac{1}{\sqrt{2 n}}\left(A_{1}^{T},-A_{1}^{T}\right)$. Considering only the treatment combinations in the design, we have that $\Lambda_{f}=\frac{1}{2 n}\left[\begin{array}{cc}I & -I \\ -I & I\end{array}\right]$. Then we get $B_{m f} \Lambda_{f} B_{m f}^{T}=$ $\frac{1}{n} \frac{1}{2 n} 4 A_{1}^{T} A_{1}=\frac{1}{n} I_{k}$. If we now use $B_{m c}$ and the $\Lambda$ matrix for the complete factorial we see that $C_{m}=\frac{n}{2^{k}} \frac{1}{n} I_{k}=\frac{1}{2^{k}} I_{k}$. Thus $\operatorname{det}\left(C_{m}\right)=\left[\frac{1}{2^{k}}\right]^{k}$ and so this design has a $D$-efficiency of $100 \%$.

Next suppose that the set of binary equations that define the OMEP, $A$, say, has at least one equation with an odd number of coefficients. Thus there are no foldover pairs in the experiment and the pairs are given by $(A,-A)$ and the argument proceeds as above.

Example 1 Let $k=4$ and consider the OMEP in Table 2(b). The pairs derived from Construction 1 and this OMEP are given in Table 3. There are only 8 of the 16 possible treatment combinations involved in these pairs but this design is as efficient as the design, based on all 8 foldover pairs, given in Table 1. If the same construction is used on the foldover from the OMEP given in Table 2(a), we get the pairs in Table 1. In other words, we get the same design as we get when we take the foldover pairs in the complete factorial.

As initially described, Construction 1 did not include the restriction that the OMEP used in the construction be regular. However Example 2 shows that without that restriction the pairs that result may not have a diagonal information matrix or be $100 \%$ efficient.

Example 2 Let $k=6$ and consider the OMEP in Table 4(a). Observe that it has one foldover pair of treatments and that it is not regular. Applying 


\begin{tabular}{|c|c|c|c|c|c|c|}
\hline$\{(0$ & 0 & 0 & 0), & (1 & 1 & 1 \\
\hline$\{(0$ & 0 & 1 & 1), & (1 & 1 & 0 \\
\hline & 1 & 0 & 1), & (1 & 0 & 1 \\
\hline & 1 & 1 & $0)$, & (1 & 0 & 0 \\
\hline
\end{tabular}

Table 3: The pairs from the OMEP with $k=4$ of resolution 4

Construction 1 to this OMEP gives 11 distinct pairs with information matrix $\frac{1}{2^{6} * 44}(48 I-4 J)$ and a D-efficiency of $97.2 \%$.

$\begin{array}{llllllllll} & & & & & & 0 & 0 & 0 & 0 \\ 0 & 0 & 0 & 0 & 0 & 0 & 1 & 1 & 1 & 1 \\ 1 & 1 & 1 & 1 & 1 & 1 & 0 & 0 & 0 & 1 \\ 0 & 1 & 0 & 1 & 1 & 0 & 1 & 1 & 1 & 0 \\ 1 & 0 & 1 & 0 & 1 & 0 & 0 & 1 & 0 & 0 \\ 0 & 1 & 1 & 1 & 0 & 0 & 1 & 0 & 1 & 1 \\ 0 & 0 & 0 & 1 & 1 & 1 & 0 & 1 & 0 & 1 \\ 0 & 0 & 1 & 0 & 1 & 1 & 1 & 0 & 1 & 0 \\ 1 & 0 & 0 & 1 & 0 & 1 & 1 & 0 & 0 & 0 \\ 1 & 1 & 0 & 0 & 1 & 0 & 1 & 0 & 0 & 0 \\ 1 & 1 & 0 & 0 & 0 & 1 & 0 & 0 & 1 & 1 \\ 0 & 1 & 1 & 0 & 0 & 1 & 0 & 0 & 1 & 1 \\ 1 & 0 & 1 & 1 & 0 & 0 & 1 & 1 & 0 & 1 \\ & & & & & 1 & 1 & 0 & 1 \\ & & \text { (a) } k=6 & & 0 & 1 & 1 & 0 \\ & & & & 0 & 1 & 1 & 0\end{array}$

(b) $k=4$

Table 4: Non-regular OMEPs of resolution 3

Construction 1 can be extended to the union of regular designs. For instance the design in Table 4(b) is the union of a $2^{4-1}$ and two copies of a $2^{4-2}$. Thus it has 8 treatment combinations in which the foldover occurs in the design and four which do not (and which are repeated). However Construction 1 applied to this design gives a set of pairs that are $100 \%$ efficient.

The second construction is found as Option 4 in Appendix A5 of Louviere, Hensher and Swait (2000).

Construction 2 Construct an OMEP with $2 k$ attributes and use the first $k$ attributes to describe the first entry in the pair and the second set of $k$ attributes to describe the second entry in the pair.

In some descriptions of this construction you are warned that main effects are only estimable if respondents are given the choice of not choosing either of 
the options. This is usually referred to as having a "neither of these" option in each pair. Even if a "neither of these" option is not required to be able to estimate main effects in a particular design, not all OMEPs of a given size are equally good, as the following example illustrates.

Example 3 Let $k=4$ and consider the OMEP in Table 5(a). This is the set of solutions to the equations $x_{2}+x_{3}+x_{4}+x_{5}=0, x_{1}+x_{3}+x_{4}+x_{6}=0$, $x_{1}+x_{2}+x_{3}+x_{7}=0$ and $x_{1}+x_{2}+x_{4}+x_{8}=0$. The pairs derived from Construction 2 are indicated by the wide column. Although all the treatment combinations appear in the pairs, four of the pairs consist of the same treatment combination repeated. This design has an information matrix for estimating main effects which is not diagonal, and a D-efficiency of about 60\%. If the pairs with repeated treatment combinations are removed then $C=\frac{1}{24} I$ and the $D$ efficiency is about 67\%. The pairs from the OMEP given by $x_{2}+x_{3}+x_{4}+x_{5}=0$, $x_{1}+x_{3}+x_{4}+x_{6}=1, x_{1}+x_{2}+x_{3}+x_{7}=1$ and $x_{1}+x_{2}+x_{4}+x_{8}=1$ again involve all 16 treatment combinations but no pair has repeated choices; see Table 5(b). This design has $C=\frac{1}{32} I$ and hence a D-efficiency of about $50 \%$. The best available design for estimating main effects is that of Table 3. It has a diagonal information matrix, uses only 4 foldover pairs and has a D-efficiency of $100 \%$. Thus none of the other designs described here is very good.

\begin{tabular}{|c|c|c|c|c|c|c|c|c|c|c|c|c|}
\hline$\{(0$ & 0 & $0),(0$ & 0 & 0 & $0)\}$ & $\{(0$ & 0 & 0 & $0), \quad(0$ & 1 & 1 & 1) $\}$ \\
\hline$\{(1$ & 0 & $0), \quad(0$ & 1 & 1 & 1) $\}$ & $\{(1$ & 0 & 0 & $0), \quad(0$ & 0 & 0 & $0)\}$ \\
\hline$\{0$ & 0 & $0),(1$ & 0 & 1 & 1)\} & $\{(0$ & 1 & 0 & $0), \quad(1$ & 1 & 0 & $0)\}$ \\
\hline (1 & 0 & $0),(1$ & 1 & 0 & 0)\} & $\{(1$ & 1 & 0 & $0),(1$ & 0 & & 1) $\}$ \\
\hline 0 & 1 & $0),(1$ & 1 & 1 & $0)\}$ & $\{(0$ & 0 & 1 & $0),(1$ & 0 & 0 & 1)\} \\
\hline (1 & 1 & $0),(1$ & 0 & 0 & 1)\} & $\{(1$ & 0 & 1 & $0),(1$ & 1 & 1 & $0)\}$ \\
\hline 1 & 1 & $0), \quad(0$ & 1 & 0 & 1) $\}$ & $\{(0$ & 1 & 1 & $0), \quad(0$ & 0 & L & $0)\}$ \\
\hline (1 & 1 & $0), \quad(0$ & 0 & 1 & $0)\}$ & $\{(1$ & 1 & 1 & $0), \quad(0$ & 1 & 0 & 1) $\}$ \\
\hline 0 & 0 & $1),(1$ & 1 & 0 & 1) $\}$ & $\{(0$ & 0 & 0 & $1),(1$ & 0 & 1 & $0)\}$ \\
\hline 0 & 0 & 1), (1 & 0 & 1 & $0)\}$ & $\{(1$ & 0 & 0 & 1), (1 & 1 & 0 & 1) $\}$ \\
\hline 1 & 0 & $1),(0$ & 1 & 1 & $0)\}$ & $\{(0$ & 1 & 0 & $1), \quad(0$ & 0 & 0 & 1)\} \\
\hline 1 & 0 & $1),(0$ & 0 & 0 & 1) $\}$ & $\{(1$ & 1 & 0 & $1), \quad(0$ & 1 & 1 & $0)\}$ \\
\hline 0 & 1 & $1),(0$ & 0 & 1 & 1) $\}$ & $\{(0$ & 0 & 1 & $1), \quad(0$ & 1 & 0 & $0)\}$ \\
\hline 0 & 1 & $1),(0$ & 1 & 0 & $0)\}$ & $\{(1$ & 0 & 1 & $1), \quad(0$ & 0 & 1 & 1) \\
\hline (0 & 1 & 1), (1 & 0 & 0 & $0)\}$ & $\{(0$ & 1 & 1 & 1), (1 & 1 & 1 & 1) \\
\hline 1 & 1 & 1), (1 & 1 & 1 & 1) $\}$ & $\{(1$ & 1 & 1 & $1),(1$ & 0 & 0 & 0) \\
\hline
\end{tabular}

(a)

(b)

Table 5: Two OMEPs with $k=8$

The reason that the designs derived from Construction 2 are so variable in performance is that we only know something about the $2 k$ attributes, rather than about each set of $k$ attributes. In the next section we will describe a 
construction for small sets of choice pairs to estimate main effects and two factor interactions in which a fixed number of attributes are different between the options in each pair in the choice set or which are unions of such sets. We believe that this construction overcomes some of the difficulties that we have identified above.

\section{Constant Difference Pairs}

Consider a regular fractional factorial design of resolution 5, F say. Choose any treatment combination not in the fraction, e say, and form pairs by pairing $\mathbf{f} \in F$ with $\mathbf{f}+\mathbf{e}$, where the addition is done component-wise modulo 2 . We will write the complete set of pairs as $(F, F+\mathbf{e})$. We refer to e as the generator of the pairs.

To evaluate the information matrix of these pairs we need to define two incidence matrices, $D_{m, e}$ and $D_{t, e}$. We define a diagonal matrix $D_{m, e}$ by $\left(D_{m, e}\right)_{X X}=1$ if $e_{X}=0$ and $\left(D_{m, e}\right)_{X X}=-1$ if $e_{X}=1$, where the attributes label the rows and columns of $D_{m, e}$. We can define a diagonal matrix $D_{t, e}$ of size $k(k-1) / 2$ by $\left(D_{t, e}\right)_{X Z, X Z}=1$ if $e_{X}=e_{Z}$ and $\left(D_{t, e}\right)_{X Z, X Z}=-1$ if $e_{X} \neq e_{Z}$ (where we label the rows and columns of $D_{t, e}$ by the ordered pairs of attributes).

We then have the following result.

Lemma 1 Consider only the treatment combinations involved in the pairs (F, $F+\mathbf{e})$. Then the information matrix, $C_{e}$, say, for these pairs is given by

$$
C_{e}=\frac{1}{4 n}\left[\begin{array}{cc}
2 I_{k}-2 D_{m, e} & 0 \\
0 & 2 I_{k(k-1) / 2}-2 D_{t, e}
\end{array}\right]
$$

Proof Let $B_{m}$ be the normalised contrast matrix for main effects associated with $F$ and let $B_{t}$ be the normalised contrast matrix for two-factor interactions associated with $F$. Then, assuming there are $k$ attributes, we know that $B_{m} B_{m}^{T}=I_{k}, B_{t} B_{t}^{T}=I_{k(k-1) / 2}$ and $B_{m} B_{t}^{T}=0$.

For convenience we order the treatment combinations in the paired comparison experiment as $\mathbf{f}_{\mathbf{1}}, \mathbf{f}_{\mathbf{2}}, \ldots, \mathbf{f}_{\mathbf{n}}$ (for some fixed but arbitrary order) followed by $\mathbf{f}_{\mathbf{1}}+\mathbf{e}, \mathbf{f}_{\mathbf{2}}+\mathbf{e}, \ldots, \mathbf{f}_{\mathbf{n}}+\mathbf{e}$. Then, considering only the treatment combinations in the pairs,

$$
\Lambda=\frac{1}{4 n}\left[\begin{array}{cc}
I & -I \\
-I & I
\end{array}\right]
$$

To calculate the information matrix we need to calculate the $B$ matrix for the pairs. If a particular attribute, $X$ say, has a 0 in $\mathbf{e}$ then the $X$ contrast in $F+\mathbf{e}$ is the same as it is in $F$ whereas if attribute $X$ has a 1 in e then the $X$ contrast in $F+\mathbf{e}$ is the negative of the one in $F$. So the matrix for main effect contrasts is given by $\left[\begin{array}{cc}B_{m} & D_{m, e} B_{m}\end{array}\right]$.

Similarly consider two attributes $X$ and $Z$. If $e_{X}=e_{Z}$ then the two factor interaction contrast for the attributes $X$ and $Z$ is the same in $F+\mathbf{e}$ as it is in 
$F$. If $e_{X} \neq e_{Z}$ then the two factor interaction contrast for attributes $X$ and $Z$ is the negative in $F+\mathbf{e}$ of the one it is in $F$. So the matrix for two factor interaction contrasts is given by $\left[\begin{array}{ll}B_{t} & D_{t, e} B_{t}\end{array}\right]$.

Then, ignoring treatment combinations not in the pairs for the time being,

$$
\begin{aligned}
C_{e} & =\frac{1}{4 n}\left[\begin{array}{cc}
B_{m} & D_{m, e} B_{m} \\
B_{t} & D_{t, e} B_{t}
\end{array}\right]\left[\begin{array}{cc}
I & -I \\
-I & I
\end{array}\right]\left[\begin{array}{cc}
B_{m}^{T} & B_{t}^{T} \\
B_{m}^{T} D_{m, e}^{T} & B_{t}^{T} D_{t, e}^{T}
\end{array}\right] \\
& =\frac{1}{4 n}\left[\begin{array}{cc}
2 I_{k}-2 D_{m, e} & 0 \\
0 & 2 I_{k(k-1) / 2}-2 D_{t, e}
\end{array}\right]
\end{aligned}
$$

as required.

Thus $C_{e}$ is diagonal and the non-zero entries in $C_{e}$ correspond to those positions in e where there is a 1 , for the "main effects" part of $C_{e}$ and to those positions, in the two factor interaction part, where one attribute corresponds to a 1 and one to a 0 in $\mathbf{e}$.

Consider the following example.

Example 4 Let $k=5$ and let $F$ be the solutions to $x_{1}+x_{2}+x_{3}+x_{4}+$ $x_{5}=0$. Let $e=(00111)$. Then the $B$ matrix for the pairs $(F, F+e)$ is the $B$ matrix for the complete factorial. If we order the treatments in $F$ as 00000, 00011, 00101, 00110, 01001, 01010, 01100, 01111, 10001, 10010, 10100, $10111,11000,11011,11101,11110$ then the contrast, within $F$, for the main effect of the first attribute is $(-1,-1,-1,-1,-1,-1,-1,-1,1,1,1,1,1,1,1,1)$. If we then add $\mathbf{e}$ to each treatment combination in $F$ we see that the contrast, within $F+\mathbf{e}$, for the main effect of the first attribute is exactly the same as that in $F$. However the contrast for the main effect of the third attribute is $(-1,-1,1,1,-1,-1,1,1,-1,-1,1,1,-1,-1,1,1)$ in $F$ and is $(1,1,-1,-1,1,1,-1,-1,1,1,-1,-1,1,1,-1,-1)$ in $F+\mathbf{e}$.

Lemma $\mathbf{2}$ Consider the pairs generated by $\mathbf{e}$ and $\mathbf{g}$ where $\mathbf{e}, \mathbf{g} \notin F$ but where $\mathbf{e}+\mathbf{g} \in F$. Then the information matrix, $C_{e, g}$, say, for these pairs is given by

$$
C_{e, g}=\frac{1}{8 n}\left[\begin{array}{cc}
4 I_{k}-2 D_{m, e} 2 D_{m, g} & 0 \\
0 & 4 I_{k(k-1) / 2}-2 D_{t, e}-2 D_{t, g}
\end{array}\right] .
$$

Proof As $\mathbf{e}+\mathbf{g} \in F$, no further treatment combinations have been used in the construction of the additional pairs. Hence we can write, again suppressing rows and columns corresponding to treatments not in any pair,

$$
\Lambda_{F e g}=\frac{1}{8 n}\left[\begin{array}{cc}
2 I & -I-P \\
-I-P & 2 I
\end{array}\right]
$$

where $P$ is a permutation matrix that ensures that $\Lambda_{F e g}$ contains the correct pairs. Consider $C=\left(B \Lambda B^{T}\right)$ and consider it as a $2 \times 2$ block matrix. Then similar calculations to those in Lemma 1 give $\left(B \Lambda B^{T}\right)_{12}=-D_{m, e} B_{m} P B_{t}^{T}-$ $B_{m} P B_{t}^{T} D_{t, e}^{T}$. If we define $D_{m, g}$ and $D_{t, g}$ for the generator $\mathrm{g}$ in the same way 
we defined $D_{m, e}$ and $D_{t, e}$ for the generator $\mathbf{e}$ then we have that $B_{m} P=$ $D_{m, e} D_{m, g} B_{m}$ since $B_{m} P$ is a permutation of the columns of $B_{m}$ and can be thought of as a permutation of the treatment combinations in $F . P$ is a permutation of the treatments in $F+\mathbf{e}$ so that the order of the treatments corresponds to that of $F+\mathbf{g}$. Since $F=F+\mathbf{e}+\mathbf{g}$ we see that the contrast matrix for the treatments in this order is $D_{m, e+g} B_{m}=D_{m, e} D_{m, g} B_{m}$, giving the result. Thus $\left(B \Lambda B^{T}\right)_{12}=0$. Again we find that $\left(B \Lambda B^{T}\right)_{11}=$ $\frac{1}{8 n}\left(4 I_{k}-2 D_{m, e}-2 D_{m, g}\right)$. Finally noting that $B_{t} P=D_{t, e} D_{t, g} B_{t}$ we see that $\left(B \Lambda B^{T}\right)_{22}=\frac{1}{8 n}\left(4 I_{k(k-1) / 2}-2 D_{t, e}-2 D_{t, g}\right)$. Hence we see that $C$ is again diagonal and the effects that can be estimated are those that correspond to a non-zero entry in one of the generators (for main effects) and those that correspond to positions with unequal entries, for two-factor interactions.

Lemma 3 Consider the pairs generated by $\mathbf{e}$ and $\mathbf{g}$ where $\mathbf{e}, \mathbf{g} \notin F$ and where $\mathbf{e}+\mathbf{g} \notin F$. The information matrix, $C_{e, g}$, say, for these pairs is given by

$$
C_{e, g}=\frac{1}{8 n}\left[\begin{array}{cc}
4 I_{k}-2 D_{m, e}-2 D_{m, g} & 0 \\
0 & 4 I_{k(k-1) / 2}-2 D_{t, e}-2 D_{t, g}
\end{array}\right] .
$$

Proof We have pairs $(F, F+\mathbf{e})$ and $(F, F+\mathbf{g})$. Thus there are $2 n$ pairs and $n$ treatments are in 2 pairs each and $2 n$ treatments are in one pair each. From the discussion above we know that $B=\frac{1}{\sqrt{(3 n)}}\left[\begin{array}{ccc}B_{m} & D_{m, e} B_{m} & D_{m, g} B_{m} \\ B_{t} & D_{t, e} B_{t} & D_{t, g} B_{t}\end{array}\right]$ and $\Lambda=\frac{1}{8 n}\left[\begin{array}{ccc}2 I & -I & -I \\ -I & I & 0 \\ -I & 0 & I\end{array}\right]$. Evaluating the information matrix for these treatment combinations only, we get that

$$
C_{F}=\frac{1}{8 n}\left[\begin{array}{cc}
4 I_{k}-2 D_{m, e}-2 D_{m, g} & 0 \\
0 & 4 I_{k(k-1) / 2}-2 D_{t, e}-2 D_{t, g}
\end{array}\right] .
$$

Including all treatments we get $C=\frac{8 n}{2^{k}} C_{F}$ as we had before.

Finally we need to consider generators that come from $F$. In this case we can use such a generator to define a fraction of $F$ and use one of the results that we have given above on that smaller fraction.

Thus whether or not the generators, or their sum, is in $F$ does not have any bearing on the estimability properties of main effects and two-factor interactions.

Lemma 4 Consider a set generators such that

- for each attribute there is at least one generator with a 1 in the corresponding position, and

- for any two attributes there is at least one generator in which the corresponding positions have a 0 and a 1 .

Then all main effects and two-factor interactions will be estimable from the pairs generated by this set of generators. 
Proof From Lemma 2 and Lemma 3 we see that diagonal entries in $C$ will be 0 only if all the $D$ matrices have entries of 1 in some position. For main effects this happens only if none of the generators have a 1 for that attribute. For two-factor interactions this happens only if all of the generators have the same entry for two attributes. But the properties of the generators given in the statement of the lemma preclude these situations from arising.

We will define an estimable set of generators to be one which satisfies the conditions of Lemma 4.

In Construction 1 we have shown that using a regular OMEP and the single generator $(1,1, \ldots, 1)$ gives designs that are $100 \%$ efficient for estimating main effects. Thus we only consider pairs for estimating main effects and two factor interactions in the remainder of this paper.

We would like to find a minimum set of generators from which all main effects and two-factor interactions can be estimated. For the estimation of main effects and two-factor interactions in the complete factorial, generators of weight $\frac{k+1}{2}$ have been shown to be optimal for odd $k$. For even $k$, generators of weights $\frac{k}{2}$ and $\frac{k}{2}+1$ have been shown to be optimal. We choose generators with these weights below, although we do not know that these weights are optimal in this setting.

In the next result we give a recursive construction for sets of generators with $\frac{k+1}{2}$ non-zero entries if $k$ is odd and $\frac{k}{2}$ non-zero entries if $k$ is even. A similar result appears in Roberts (2000).

Lemma 5 If $2^{m} \leq k<2^{m+1}$ then there is a estimable set with $m+1$ generators.

Proof The proof proceeds recursively once we have the first two cases. When $k=2$ use the generators $(1,0)$ and $(0,1)$. When $k=3$ use the generators $(1,1$, $0)$ and $(0,1,1)$.

For the recursive construction it is advantageous to write the generators as two sets, those positions in which the generator contains a 1 and those positions in which the generator contains a 0 . Hence we get the partitions $(\{(1),(2)\}$, $\{(2),(1)\})$ for $k=2$ and $(\{(1,2),(3)\},\{(2,3),(1)\})$ for $k=3$.

Consider $k=2 s+1$. We write the first partition as $\{(1,2, \ldots, s+1),(s+$ $2, s+3, \ldots, 2 s+1)\}$. We then partition the sets of size $s$ and $s+1$ and take the union of the first set in the first partition of each and the union of the second set in the first partition of each to get the second partition for $k=2 s+1$. We continue in this way to get all the partitions for $k=2 s+1$. The only time that this might not work is when $s+1$ is a power of 2 since in that case $s+1$ has one more partition than $s$. However using the initial generators given above we see that the final generator for a power of 2 is just the foldover of the second last generator. This generator is required only so that main effects can be estimated, not for the estimation of two-factor interactions, and all the main effects for the first $s+1$ attributes can be estimated from the first generator. Hence we can ignore this generator when doing the recursive construction. This completes the construction for odd $k$. 
Consider $k=2 s$. Do exactly the same construction as for odd $k$, using an initial partition of $\{(1,2, \ldots, s),(s+1, s+3, \ldots, 2 s)\}$.

This proof is completed by noting that the set of generators satisfies the conditions of Lemma 4.

Example 5 Let $k=8$. Then the partition that arises is $(\{(1,2,3,4),(5,6,7,8)\}$, $\{(1,2,5,6),(3,4,7,8)\},\{(1,3,5,7),(2,4,6,8)\},\{(2,4,6,8),(1,3,5,7)\})$. The final partition is only required to ensure that the main effect of attribute 8 can be estimated. Now construct the partition for $k=15$. We get $(\{(1,2,3,4,5,6,7,8)$, $(9, A, B, C, D, E, F)\},\{(1,2,3,4,9, A, B, C),(5,6,7,8, D, E, F)\},\{(1,2,5,6,9, A, D, E)$, $(3,4,7,8, B, C, F)\},\{1,3,5,7,9, B, E, F),(2,4,6,8, A, C, D)\})$ and the final partition from $k=8$ is not required since the main effect of attribute 8 can be estimated because there is a 1 in position 8 in the first generator.

The efficiency of the designs that result from the lemma above depend on the particular resolution 5 design that is used; see Table 6 .

For odd $k$ it is possible to use balanced incomplete block designs (BIBD) to give a set of generators in which each main effect and each interaction effect is estimated using the same number of generators. (Recall that a balanced incomplete block design is a collection of $b k$-sets, or blocks, of a $v$-set such that each element of the $v$-set appears in exactly $r$ blocks and any pair of elements appears in $\lambda$ blocks. We write $(v, b, r, k, \lambda)$ BIBD. If $v=b$ then $r=k$ and the design is said to be symmetric (SBIBD), written $(v, k, \lambda)$. Realise that it is the $v$ of the BIBD setting that is the $k$ of the choice set setting.) The pairs that result from Lemma 6 are often very efficient and relatively small. However there does not appear to be a general expression for the efficiency of these designs.

Lemma 6 1. The blocks of a $(4 t+3,2 t+2, t+1) S B I B D$ can be used to give $4 t+3$ generators, each with weight $2 t+2$, such that each main effect and each two factor interaction can be estimated from $2 t+2$ of the generators.

2. The blocks of a $(4 t+1,2(4 t+1), 2(2 t+1), 2 t+1, t+1)$ BIBD can be used to give $2(4 t+1)$ generators, each with weight $2 t+1$, such that each main effect can be estimated from $4 t+2$ of the generators and each two factor interaction can be estimated from $6 t+2$ of the generators.

3. For even $k$, estimable sets with high efficiency can be found by deleting one treatment from the designs above.

Proof The results follow by counting the number of blocks with one specific treatment, for main effects, and the number of blocks with only one of two specific treatments, for interaction effects.

In the following table we give some fractions and generators, the number of pairs in the choice set and the $D$-efficiency of the set of pairs. For each value of $k$, the set of generators that come from Lemma 5 are indicated by an * and those that come from Lemma 6 are indicated by $\dagger$. In Table 6 we use $I \equiv A B C D E$, 
say, to represent the solutions to the equation $x_{1}+x_{2}+x_{3}+x_{4}+x_{5}=0$ and $I \equiv A B C D E \equiv D E F G H$ to represent the solutions to both the equations $x_{1}+x_{2}+x_{3}+x_{4}+x_{5}=0$ and $x_{4}+x_{5}+x_{6}+x_{7}+x_{8}=0$. In the table $M R$ refers to Mathon and Rosa (1996).

The designs for $k=4$ with 48 pairs and with 32 pairs are equivalent to designs given in van Berkum (1987b, Chapter 5). The method of construction is quite different, however, as he focuses on finding sets of pairs within one or more fractions and we specify the fraction and one, or more, generators for the pairs. For $k=5$ for example, van Berkum's design with 80 pairs has an efficiency of $84 \%$ compared to our design with 80 pairs with an efficiency of $96.5 \%$.

Table 6: $D$-efficiency and number of pairs for some constant difference choice pairs

\begin{tabular}{|c|c|c|c|c|}
\hline $\mathrm{k}$ & $\mathrm{F}$ & generators & $\begin{array}{l}\text { number } \\
\text { of pairs }\end{array}$ & $\begin{array}{l}\text { efficiency } \\
\quad(\%)\end{array}$ \\
\hline $3 \dagger$ & complete & $011,101,110$ & 12 & 100 \\
\hline $3^{*}$ & complete & 011,101 & 8 & 94.5 \\
\hline 4 & complete & all vectors of weights 2 and 3 & 80 & 100 \\
\hline 4 & complete & $\begin{array}{l}\text { omit any one weight } 2 \\
\text { or weight } 3 \text { vector }\end{array}$ & 72 & 99.6 \\
\hline 4 & complete & $\begin{array}{l}\text { omit any two weight } 2 \text { vectors } \\
\text { or any two weight } 3 \text { vectors }\end{array}$ & 64 & 99.21 \\
\hline 4 & complete & $\begin{array}{l}\text { six weight } 2 \text { vectors and } \\
\text { any weight } 3 \text { vector }\end{array}$ & 56 & 98.95 \\
\hline $4 \dagger$ & complete & $\begin{array}{l}1100,1010,1001 \\
0110,0101,0011\end{array}$ & 48 & 99.03 \\
\hline 4 & complete & $1110,1101,1011,0111$ & 32 & 98.01 \\
\hline $4^{*}$ & complete & $1100,1010,0101$ & 24 & 93.98 \\
\hline $5 \dagger$ & $I \equiv A B C D E$ & all weight 3 vectors & 160 & 100 \\
\hline 5 & $I \equiv A B C D E$ & $\begin{array}{c}\text { any nine weight } 3 \\
\text { vectors }\end{array}$ & 144 & 99.60 \\
\hline 5 & $I \equiv A B C D E$ & $\begin{array}{c}\text { any eight weight } 3 \\
\text { vectors }\end{array}$ & 128 & 99.08 \\
\hline 5 & $I \equiv A B C D E$ & $\begin{array}{c}\text { 11100, 10011, 10101, } \\
\text { 11010, 01110, 00111, } \\
11001\end{array}$ & 112 & 98.46 \\
\hline 5 & $I \equiv A B C D E$ & $\begin{array}{l}11100,10110,10101 \\
11010,11001,10110\end{array}$ & 96 & 97.92 \\
\hline 5 & $I \equiv A B C D E$ & $\begin{array}{c}11100,10110,10101, \\
11010,11001\end{array}$ & 80 & 96.49 \\
\hline 5 & $I \equiv A B C D E$ & $\begin{array}{c}11100,10110,10101 \\
11010\end{array}$ & 64 & 95.72 \\
\hline $5^{*}$ & $I \equiv A B C D E$ & $11100,11010,01101$ & 48 & 91.32 \\
\hline
\end{tabular}




\begin{tabular}{|c|c|c|c|c|}
\hline $6 \dagger$ & complete & $\begin{array}{c}10100,111010,011101, \\
001110,100111,010011, \\
101001\end{array}$ & 224 & 100 \\
\hline 6 & complete & any 6 of the generators above & 192 & 98.98 \\
\hline 6 & complete & any 5 of the generators above & 160 & 97.44 \\
\hline 6 & complete & $\begin{array}{l}\text { the four weight } 3 \text { vectors } \\
\text { from above }\end{array}$ & 128 & 96.61 \\
\hline $6 \dagger$ & $I \equiv A B C D E F$ & $\begin{array}{c}\text { 110100, 111010, 011101, } \\
\text { 001110, 100111, 010011, } \\
101001\end{array}$ & 176 & 99.46 \\
\hline 6 & $I \equiv A B C D E F$ & $\begin{array}{l}\text { 111100, 001111, 100111, } \\
111010,111001,010111\end{array}$ & 96 & 95.71 \\
\hline 6 & $I \equiv A B C D E F$ & $\begin{array}{c}\text { 111100, 001111, 100111, } \\
\text { 111010, 111001 }\end{array}$ & 80 & 93.75 \\
\hline 6 & $I \equiv A B C D E F$ & $\begin{array}{l}111100,001111,100111, \\
111010\end{array}$ & 64 & 92.49 \\
\hline $6^{*}$ & $I \equiv A B C D E F$ & $111000,001011,100110$ & 96 & 91.85 \\
\hline $7 \dagger$ & $I \equiv A B C D E F G$ & $\begin{array}{c}1110100,0111010,0011101, \\
1001110,0100111,1010011, \\
1101001\end{array}$ & 224 & 100 \\
\hline 7 & $I \equiv A B C D E F G$ & any 6 of these 7 vectors & 192 & 98.98 \\
\hline 7 & $I \equiv A B C D E F G$ & any 5 of these 7 vectors & 160 & 97.44 \\
\hline $7^{*}$ & $I \equiv A B C D E F G$ & $1111000,1100110,1010011$ & 96 & 91.85 \\
\hline $8^{*}$ & $\begin{array}{l}I \equiv A B C D E \\
\equiv D E F G H\end{array}$ & $\begin{array}{c}11110000,11001100,10101010, \\
01010101\end{array}$ & 256 & 92.96 \\
\hline 81 & $\begin{array}{l}I \equiv A B C D E \\
\equiv D E F G H\end{array}$ & $\begin{array}{c}\text { generators from first } \\
(9,18,10,5,5) \text { in } M R\end{array}$ & 1120 & 99.97 \\
\hline $8 \dagger$ & $\begin{array}{l}I \equiv A B C D E \\
\equiv D E F G H\end{array}$ & $\begin{array}{c}\text { generators from sixth } \\
(9,18,10,5,5) \text { in } M R\end{array}$ & 1120 & 99.88 \\
\hline 81 & $\begin{array}{l}I \equiv A B C D E \\
\equiv D E F G H\end{array}$ & $\begin{array}{c}\text { generators from } 11 \text { th } \\
(9,18,10,5,5) \text { in } M R\end{array}$ & 1056 & 99.90 \\
\hline $8^{*}$ & $\begin{aligned} I & \equiv A B C D E F \\
& \equiv D E F G H\end{aligned}$ & $\begin{array}{c}11110000,11001100,10101010, \\
01010101\end{array}$ & 224 & 86.51 \\
\hline $8 \dagger$ & $\begin{aligned} I & \equiv A B C D E F \\
& \equiv D E F G H\end{aligned}$ & $\begin{array}{c}\text { generators from first } \\
(9,18,10,5,5) \text { in } M R\end{array}$ & 992 & 99.81 \\
\hline 81 & $\begin{aligned} I & \equiv A B C D E F \\
& \equiv D E F G H\end{aligned}$ & $\begin{array}{c}\text { generators from sixth } \\
(9,18,10,5,5) \text { in } M R\end{array}$ & 960 & 99.68 \\
\hline $8 \dagger$ & $\begin{aligned} I & \equiv A B C D E F \\
& \equiv D E F G H\end{aligned}$ & $\begin{array}{c}\text { generators from } 11 \text { th } \\
(9,18,10,5,5) \text { in } M R\end{array}$ & 1056 & 99.9 \\
\hline
\end{tabular}

\section{Concluding Remarks}

The results in Section 4 can be used to construct designs in which each attribute has a number of levels equal to a power of two. Broadly one does this by replac- 
ing each attribute with an appropriate number of pseudo-factors and ensuring that all interactions between pseudo-factors associated with the same original attribute can be estimated in the proposed design.

For example, to construct a design to estimate main effects for four attributes, three with two levels and one with four levels, construct a design for five attributes, $A, B, C, D$ and $E$, say, and ensure that the interaction between $D$ and $E$ is also estimable. Thus the generators $(1,1,1,1,1),(1,1,1,1,0)$ and $(1,1,1,0,1)$ give 48 pairs from which all main effects in the original attributes can be estimated. This set of pairs appears to be optimal for this situation.

To construct a design to estimate main effects for three attributes, one with two levels and two with four levels, we again construct a design using $A, B$, $C, D$ and $E$ but now the interaction between $B$ and $C$ must also be estimable. The generators of the previous paragraph will not work since this interaction is not estimable. Instead use the generators $(1,1,1,1,1),(1,1,0,1,0)$ and $(1,0,1,0,1)$. Again this appears to be the optimal set of pairs for this setting. If these generators are used in the previous paragraph, then they have a $D$-efficiency of $\frac{4}{9}$ relative to the generators given in the previous paragraph.

To use these ideas for attributes with 8 levels will require that three factor interactions be estimable in the design involving the pseudo-factors.

In this paper we have used $D$-efficiency to compare various designs. We have not given any consideration to task complexity. If more attributes differ between options in a choice set does the task of choosing become more complex and is respondent consistency affected? Designs constructed using the methods of Section 4 have been used to investigate these questions in Severin (2000).

Acknowledgement We thank a referee for detailed comments which improved the presentation of the paper. The work described in this paper was supported by the Australian Research Council with grant A79906045.

\section{References}

Berkum, E.E.M. van (1987a). Optimal paired comparison designs for factorial and quadratic models. Journal of Statistical Planning and Inference 15, 265278.

Berkum, E.E.M. van (1987b). Optimal paired comparison designs for factorial experiments. CWI Tract 31, Amsterdam.

Berkum, E.E.M. van (1989). Reduction of the number of pairs in paired comparison designs and exact designs for quadratic models. Computational Statistics and Data Analysis 8, 93-107.

El-Helbawy, A.T., Ahmed, E.A. and Alharbey, A.H. (1994). Optimal designs for asymmetrical factorial paired comparison experiments, Communications in Statistics - Simulation 23, 663-681.

Grossman, H., Holling, H. and Schwabe, R. (2002). Adavances in optimum experimental design for conjoint analysis and discrete choice models, Econometric 
Models in Marketing 16, 91-115.

Kuhfeld, Warren F., Multinomial Logit, Discrete Choice Modelling, SAS Institute, 2000. Available at http://ftp.sas.com/techsup/download/technote/ts621.pdf

Louviere, Jordan J. (1988). Analyzing Decision Making: Metric Conjoint Analysis. Sage University Paper series on Quantitative Applications in the Social Sciences, series no. 67. Beverley Hills: Sage Publications.

Louviere, Jordan J., Hensher, David A. and Swait, Joffre D. (2000). Stated Choice Methods: Analysis and Application. Cambridge: Cambridge University Press.

Louviere, J.J. and Woodworth, G. (1983). Design and analysis of simulated consumer choice or allocation experiments: An approach based on aggregated data. Journal of Marketing Research 20, 350-367.

Mathon, Rudolf and Rosa, Alexander (1996). 2- $(v, k, \lambda)$ designs of small order, in Handbook of Combinatorial Designs, (CJ Colbourn and J Dinitz, Eds), Boca Raon: CRC Press, 3-41.

Offen, W.W. and Littell, R.C. (1987). Design of paired comparison experiments when treatments are levels of a single quantitative variable. Journal of Statistical Planning and Inference15, 331-346.

Roberts, Ian, (2000). Personal communication.

Severin, Valerie C., Comparing Statistical Efficiency and Respondent Efficiency in Choice Experiments, PhD thesis, University of Sydney, 2000.

Street, Deborah J., Bunch, David S. and Moore, Beverley, (2001). Optimal designs for $2^{k}$ paired comparison experiments, Communications in Statistics Theory and Methods 30, 2149-2171. 